\title{
Role of Radiotherapy in Locally Advanced, Fungating, and Inoperable Breast Cancer
}

\author{
Pramod Kumar Singh ${ }^{1} \quad$ jitendra Kumar Verma ${ }^{1}$ Waseem Raza ${ }^{1} \quad$ Sanjay Kumar Verma ${ }^{1} \quad$ Nitin Kumar ${ }^{1}$ \\ ${ }^{1}$ Department of Radiotherapy and Oncology, J.K. Cancer Institute, \\ Kanpur, Uttar Pradesh, India \\ Address for correspondence Pramod Kumar Singh, MD, Department \\ of Radiotherapy and Oncology, J.K. Cancer Institute, Kanpur 208002, \\ Uttar Pradesh, India (e-mail: drpramodsingh16@gmail.com).
}

\begin{abstract}
Keywords

- breast cancer

- chemotherapy

- palliative

- radiotherapy

Introduction In India, majority of patients are diagnosed with breast cancer in locally advanced stage. Surgery, chemotherapy, and radiotherapy (RT) are the mainstay of treatment for many patients who are not fit for surgery and need local RT.

Materials and Methods This was a single-center, nonstratified, single-blind, nonplacebo-controlled parallel group intervention study with imbalanced randomization done at our institute. Adult patients of 30 to 65 years of age with histologically proven carcinoma of breast with biopsy were registered and assessed.

Results In total, 30 patients were included in the study and the mean age was 50 years. All the patients received $30 \mathrm{~Gy} / 10$ fractions in 2 weeks and the response was assessed after 4 weeks of completion of RT. Twelve patients observed more than $70 \%$ reduction in tumor size and good subjective relief, nine patients observed $40 \%$ reduction in tumor size, and six patients observed $30 \%$ reduction in tumor size. Two patients did not show any response to RT, while one patient did show progress. All patients received chemotherapy before $\mathrm{RT}$ and 28 patients received chemotherapy after $\mathrm{RT}$.

Conclusion Thus, whether it is $20 \mathrm{~Gy}$ in 5 fractions or $30 \mathrm{~Gy}$ in 10 fractions is debatable and needs further study of larger group to establish their benefit on each other. However, $30 \mathrm{~Gy}$ in 10 fractions gives good subjective response to patients and improves the quality of life.
\end{abstract}

\section{Introduction}

Patients who present with locally advanced breast cancer are at risk of both distant and locoregional disease recurrence. Optimal treatment of locally advanced breast cancer requires a multidisciplinary approach that incorporates diagnostic imaging, chemotherapy, appropriate surgical intervention, radiation, and, if indicated, biological and hormonal therapies. The treatment outcome of an individual patient may depend on the degree to which this multidisciplinary approach is integrated and the expertise of the treatment team. This is especially important in the management of patients with locally advanced disease because the definition of optimal breast cancer treatment for such patients is constantly evolving and such patients have the highest risk of disease recurrence without optimal treatment and require

DOI https://doi.org/ 10.1055/s-0040-1702907 ISSN 2454-6798. the most complex decision making. The outcome of patients who have locally advanced breast cancer has improved dramatically over the past 30 years. Before the routine use of chemotherapy, most patients were treated with mastectomy, radiation, or a combination of the two; yet despite these approaches, most would develop distant metastases and die due to this disease. The introduction of progressively more effective chemotherapy and hormone therapy regimens has significantly improved the prognosis. As additional improvements in systemic treatments further reduce the chance of dying from metastatic disease, the complete eradication of locoregional disease becomes increasingly more important. This study focuses on the management of locally advanced breast cancer, with a particular focus on locoregional control and radiation therapy. Most commonly, this term refers to stage III disease, meaning advanced primary or nodal disease 
with or without clinically evident systemic metastases. Inflammatory breast cancer is an important subcategory of locally advanced breast cancer that has a unique epidemiology, presentation, and biology. Inflammatory breast cancers are rare, accounting for only $2 \%$ of all breast cancers. ${ }^{1}$ During the 1990s, the incidence of inflammatory breast cancer increased slightly. No known risk factors have been identified that are unique for the development of this form of breast cancer. However, the disease tends to occur in a younger population than does noninflammatory breast cancer. The outcome of patients who present with locally advanced breast cancer was once poor, but improvements in treatments have changed the prognosis considerably. Tumor stage increases the risk of spread through the lymphatics to involve regional lymph nodes and/or spreads hematogenously to involve distant sites such as the liver, lung, bone, and brain. Most advanced primary tumors are associated with axillary lymph node involvement at the time of diagnosis, a Rotter's lymph node, and an internal mammary lymph node. The clinical course of locally advanced breast cancer depends on several factors, including the specific disease characteristics at presentation, the biological features of the disease, and the treatment given. Without treatment, almost all locally advanced breast cancers eventually metastasize to visceral organs and become life threatening. ${ }^{2,3}$ Local disease progression can lead to ulceration of the breast skin, pain, bleeding, and infection. Progression of untreated regional lymphatic disease can cause pain, brachial plexopathy, arm edema, obstruction and thrombosis of the brachial vasculature, and skin ulceration. Treatment advances have improved both survival times and survival rates for women with locally advanced breast cancer. Before the use of systemic treatment became routine, patients with advanced disease were treated with mastectomy, radiation therapy, or both and had 5-year survival rates of only 25 to $45 \%{ }^{4}$ Currently, 5 -year survival rates benefit $80 \%$ for patients with stage IIIA disease and $45 \%$ for patients with stage IIIB disease when treated with surgery, radiation therapy, and chemotherapy. ${ }^{5}$ When assessing the survival of patients with stage III breast cancer, it is important to appreciate the heterogeneity of stage III disease. Another important aspect to consider when evaluating the outcome of stage III breast cancer over time is the effect of stage migration. Improvements in diagnostic imaging increase the likelihood of detecting metastatic disease and thereby result in reclassifying some cases of stage III disease as stage IV. Locally advanced breast cancer most commonly is diagnosed after a palpable mass is detected within the breast. Advanced disease can cause symptoms such as locoregional pain, bleeding, paresthesia, and paresis. As previously indicated, it is critically important to determine the onset of symptoms and the rate of disease progression to reach an accurate diagnosis as to whether an advanced breast cancer represents an inflammatory carcinoma.

\section{Materials and Methods}

Thirty patients were analyzed in study between 2016 and 2018. All patients were registered in J.K. Cancer Institute. They were locally advanced and received systemic chemotherapy, on progression of local disease. Patients received local radiotherapy (RT) including regional drainage area. All patients were planned with two tangential fields and one supraclavicular and axillary field. All patients were assessed after 4 weeks of completion of RT. All patients assessed response after completion of RT by RESCIT (Response Evaluation Criteria in Solid Tumors) criteria and also subjective and objective response.

\section{Patients' Characteristics}

Patients' characteristics are summarized in - Table 1. Patients registered between January 2016 and December 2018 were analyzed. The median age was 52 years. The median duration of follow-up was 6 months. Bleeding from local site was most common presenting symptoms followed by discomfort and fever followed by foul-smelling discharge.

Table 1 Patients' characteristics

\begin{tabular}{|l|l|}
\hline Total patients & 30 \\
\hline Age (y) & \\
\hline $30-40$ & 9 \\
\hline $41-50$ & 10 \\
\hline $51-60$ & 7 \\
\hline $61-70$ & 4 \\
\hline KPS & \\
\hline $70-80$ & 14 \\
\hline$>80$ & 16 \\
\hline Histopathology & \\
\hline Infiltrating duct carcinoma & 24 \\
\hline Adenocarcinoma & 5 \\
\hline Lobular carcinoma & 1 \\
\hline Stage & \\
\hline III & 16 \\
\hline IV & 14 \\
\hline Chemotherapy & \\
\hline 6 cycles & 16 \\
\hline More than 6 cycles & 14 \\
\hline Surgery & \\
\hline MRM & 13 \\
\hline Lumpectomy & 3 \\
\hline No surgery & 13 \\
\hline Bilateral breast & 1 \\
\hline Receptor status & \\
\hline ER/PR positive & 3 \\
\hline ER/PR negative/HER2 positive & 2 \\
\hline ER/PR positive/HER2 negative & 16 \\
\hline ER/PR/HER2 negative & 2 \\
\hline ER/PR/HER2 positive & \\
\hline & \\
\hline
\end{tabular}

Abbreviations: ER, estrogen receptor; HER2, human epidermal receptor 2; KPS, Karnofsky Performance Status; MRM, modified radical mastectomy; PR, progesterone receptor. 


\section{Results}

Total 30 patients were included in the study and the mean age was 50 years. All the patients received $30 \mathrm{~Gy} / 10$ fractions in 2 weeks and the response was assessed after 4 weeks of completion of RT. Twelve patients observed more than 70\% reduction in tumor size and good subjective relief, nine patients observed $40 \%$ reduction in tumor size, and six patients observed $30 \%$ reduction in tumor size. Two patients did not show any response to RT, while one patient did show progress. All patients received chemotherapy before RT and 28 patients received chemotherapy after RT. Two patients did not come for further treatment. Twelve patients received hormone therapy after completion of RT and two patients develop brain metastasis who received palliative whole brain RT.

\section{Discussion}

There is an ongoing debate regarding the benefit of locoregional treatment of the breast cancer with surgery and/or radiation in women whose initial breast cancer presentation includes distant metastatic disease. The question that remains to be answered is what effect locoregional treatment of the primary tumor may have on the overall survival in addition to other measures including patient-reported quality of life. There is retrospective data that has demonstrated a survival benefit to locoregional treatment, but there is a concern that these studies have significant selection bias (i.e., younger women and/or women with better performance status may be more likely to be offered locoregional treatment). ${ }^{6}$ While this debate continues, the fact remains that many women with locally advanced or metastatic breast cancer, who have symptoms stemming from their primary breast tumor, can receive tremendous benefit from radiation therapy as a palliative treatment. There is no guideline-based radiation dose for palliation of a primary breast tumor. Common palliative regimens such as $20 \mathrm{~Gy}$ in 5 fractions or $30 \mathrm{~Gy}$ in 10 fractions may be used in this setting. The benefit of 30 to 35 Gy in 5 fractions is its higher biological equivalent dose (BED) compared with other palliative regimens. This dose regimen yields a BED equivalent to $70 \mathrm{~Gy}$ in standard fractionation (2 $\mathrm{Gy} /$ fraction), which is a dose appropriate for gross disease in the setting of breast cancer. By comparison, a schedule of 30 $\mathrm{Gy}$ in 10 fractions delivers a BED of only $60 \mathrm{~Gy}$.

\section{Conclusion}

Thus, whether it is 20 Gy in 5 fractions or 30 Gy in 10 fractions is debatable and needs further study of larger group to establish their benefit on each other. However, $30 \mathrm{~Gy}$ in fractions gives good subjective response to patients and improves the quality of life.

\section{Conflict of Interest}

None declared.

\section{References}

1 Levine PH, Steinhorn SC, Ries LG, Aron JL. Inflammatory breast cancer: the experience of the surveillance, epidemiology, and end results (SEER) program. J Natl Cancer Inst 1985;74(2):291-297

2 Haagensen CD, Cooley E. Radical mastectomy for mammary carcinoma. Ann Surg 1969;170(6):884-888

3 Haagensen CD, Stout AP. Carcinoma of the breast: criteria of inoperability. Ann Surg 1943;118:859870

4 Fracchia AA, Evans JF, Eisenberg BL. Stage III carcinoma of the breast. A detailed analysis. Ann Surg 1980;192(6):705-710

5 Hortobagyi GN, Singletany EA, Buchholz TA. Locally advanced breast cancer. In: Singletary SE, Robb GL, GN H, eds. Advanced Therapy of Breast Disease, 2nd edition. Hamilton, Ontario: B.C. Decker, Inc; 2004:498508

6 Truong PT. Local treatment of the primary tumor in patients presenting with stage IV breast cancer: a first, and what's up ahead. Int J Radiat Oncol Biol Phys 2017;97(3):443-446 